\title{
Intergenerational Experiences on Romantic Relationship: An Exploratory Study on Balinese Parents and Adolescents
}

\author{
D A Widhiyanti MP ${ }^{1}, K$ W Yuniarti ${ }^{2}$, W M Minza ${ }^{3}$ \\ ${ }^{1}$ Universitas Pendidikan Ganesha, ${ }^{1,2,3}$ Universitas Gadjah Mada \\ 1dewi.arum.w@mail.ugm.ac.id, ${ }^{2}$ kwartarini_psy@ugm.ac.id, ${ }^{3}$ wminza@ugm.ac.id
}

\begin{abstract}
Romantic relationships are experiences that become an integral part of individual development in each generation. This exploratory study uses qualitative approach aiming to gain an overview of the experiences regarding romantic relationships from Balinese parents as the first generation, and adolescents as the second generation. The method of data collection was a survey conducted by distributing questionnaires with open-ended questions. Respondents were parents aged 40-52 years old $(n=145)$ and adolescent aged 14-17 years old $(\mathrm{n}=191)$. The data was analyzed through a coding process to obtain themes that emerged in the dimensions of romantic relationships. The results showed that there were a number of both differences and similarities within the experiences of romantic relationships reported by both generations seen from the dimensions of romantic relationships, namely the dimension of involvement, relationships content, romantic partner selection, relationships quality, as well as cognitive and emotional processes. It can be concluded that the contrasts in experiences identified were caused by the influence of interpersonal contexts; in particular the shift in parenting from strict supervision to inadequate supervision with more freedom for the individuals. Other aspects included the influence of modernization through technological developments that allowed the influx of foreign culture. This also facilitated the interaction and communication of adolescents, and the similarities showing transference from the previous generation.
\end{abstract}

Keywords: Bali, Experiences, Intergenerational, Romantic Relationship

\section{INTRODUCTION}

Romantic relationships are important part of the journey of individual psychological development. Romantic relationship is defined as mutually recognized voluntary interactions, which obtains different intensities, generally characterized by expressions of affection and the possibility of current or anticipated sexual behavior, when compared to other peer relationships [1] [2]. The development of knowledge, skills, and experience needed to form romantic relationship as well as healthy and positive sexuality is a process throughout the life span [3]. Adolescents and young adults in particular, are prominent developmental periods for the exploration of romantic relationships and sexual behavior [2]. For the generation that is now parents, the romantic relationships similarly developed prominently in their adolescence period in the conditions of society and the development of modernization that are divergent from what is existing at present. 
Collins [1] proposes five dimensions of romantic relationship, namely (1) romantic participation or experience: this dimension combines several things, such as whether or not someone is dating, when (s)he starts dating, the duration of romantic relationship along with, the frequency and consistency of dating and the relationship; (2) romantic partner selection: this dimension is related to the characteristics of the individuals involved in romantic relationship; (3) relationship content: this dimension discusses what the couples do and do not do together as well as how they spend their time; (4) the quality of romantic relationship: it discusses the relative degree of supports and conflicts within the romantic relationship, as well as the positive, supportive, and profitable experiences in the relationship in comparison to the negative and potentially detrimental interactions; (5) cognitive process and dialogue: this dimension includes perceptions, attributions, and representations of individuals, couples, and relationships, as well as emotional moods generated in romantic congregations and affective agreements related to participation and dissolution of relationships.

The theories that generally function as stepping stones in current research outline the relationship in normative social experiences of childhood and adolescence. This includes a biosocial perspective; ecological perspective; and interpersonal formulations, such as proximity and interdependence theory [2]. It is more appropriate for this study to use an ecological perspective. The ecological perspective emphasizes the social and cultural context that encourages while inhibiting close relationships and gives meaning. The forms of ecological influence such as history, characteristics of social, economic, political, geographic, cultural, institutional and community aspects [2]. Values, norms, and expectations in romantic interactions form the background in which the relationship develops[4].

Markus and Kitayama [4] in their research distinguished two cultural views, namely collectivism and individualism. These major viewpoints become the direction for crosscultural research. Based on this view, Asian culture emphasizes family attachments. The romantic process of Asian adolescents pays attention to this primary connection and the inevitable progress of social network. In contrast, western culture places more emphasis on the formation of adolescent relationships outside of the family. Since a romantic relationship is a sign of maturity and pulls out the adolescents from family influence, it is reasonable to expect that Asian and western cultural norms will differ in accepting adolescence romanticism.

Every individual has some experiences in romantic relationship that varies from one another and it is inseparable from the socio-cultural context in which they are located. Balinese people, who are known to have a strong culture that is related with traditions and values of life, are also inseparable from the experience of romantic relationship that generally begins in adolescence period. However, modernization has brought changes in the society and has an impact on changing beliefs, thinking habits, etc. [5]. The concept of generation refers to a group of people who are distinguished from others based on their age. Different generations can be formed by different social experiences in the years of their formation. Different generations may have various values because of the variety of life challenges and the background of formative socialization [6].

Intergenerational differences assume that each generation, due to the fact that generations are distinguished for years apart from each other, experience different ways of life. Therefore, each generation has their own experiences and problems that differ from those faced by their parents' generation [7]. In this context, it is assumed that each age group experiences life uniquely, they are developing attitudes, beliefs, characteristics, and values that make them live their lives differently from other age groups.

The group of Balinese parents, have their own experience in romantic relationship in their adolescence period, which is assumed to have not attained the onslaught of 
modernization as it is today. On the contrary, Balinese adolescents at present, referred to as the second generation in this study, grow in the environmental conditions that have undergone many changes and developments, which are influenced by the development of information and communication technology as previously stated. The purpose of this study was to explore the experiences from the frame of romantic relationship dimensions between the two generations.

\section{RESEARCH METHOD}

This research became a part of the exploration phase on the topic of romantic relationships within Balinese, which utilized qualitative research approach. The data was collected by using a survey through the distribution of open-ended questionnaires with informed consent (as part of research ethics). The participants of the study consisted of first generation and second-generation groups. The first-generation group included participants aged 40-52 years, both male and female, who were the parents of adolescents $(n=145)$; the second-generation group was the participants of adolescent boys and girls aged 14-17 years (n = 191). Parent generation, or the first generation, completed questionnaires regarding the perceptions and experiences that compared the romantic relationship in the past and at present; the second generation filled out a questionnaire about their experiences of romantic relationships. The data was analyzed through coding stages, namely open coding, axial coding, and selective coding, to bring up themes in the dimensions of the romantic relationship.

\section{RESULTS AND DISCUSSION}

The results of data analysis were classified based on the differences of the romantic relationship between two generations. The parents' perceptions which were based on the dimensions of romantic relationships, included involvement, relationship content, romantic partner selection, relationship quality, as well as cognitive and emotional aspects.

\subsection{Involvement in romantic relationship}

The first generation reported the involvement at a more mature age when first establishing romantic relationship compared to the age when the second generation involved in the relationship. The adolescents of the second generation tended to get to know the opposite sex more closely and engage in a romantic relationship at a very young age, that was when they were still in junior high school or even in elementary school. This is quite different from the condition when the first generation used to know about dating activities which was when they approached adulthood. At that time, only few adolescents who were dating and dared to show their relationship. It is in contrast to the second-generation adolescents, most of whom knew dating with the frequency of more than once throughout their teenage years. It was easier for them to start and end their relationship and they still felt free to change romantic partners. Apart from the prohibition of parents, the first generation also reported that the desire to focus more on education and plan a future to get decent jobs due to economic reasons caused the first generation did not want to establish romantic relationship in their adolescence yet.

More than $60 \%$ of adolescent participants in this study had already been in or were in romantic relationship, and the rest had never been in a romantic relationship. From the experience reported by the participants, it was showed that the adolescents of the second generation were first in the romantic relationship at the age range of 10 to 15 years, with the 
frequency of relationship ranging from one to three times to their current age. The shortest duration of the relationship was two weeks and the longest was 3 years.

The adolescents who had never had a romantic relationship had certain reasons: the feeling of not old enough, the absence of the parents' permit, the desire to focus on education, the absence of special interest to the opposite sex, a more selective attitude in choosing romantic partners because they did not want to waste time if they chose the wrong partner. The reasons given by this group of adolescents were not much different from the reasons of the first generation who explained that the adolescents in their generation were rarely in romantic relationship. The view which believed that dating were taboo was hold by the first generation, but it did not appear and was not hold by the second generation.

The attitude of Balinese parents of the second generation that prohibited their children from dating, for the reasons of being young and encouraging them to focus on learning, did not necessarily cause the second-generation adolescents not to have romantic relationship, but on the other hand caused them to secretly date.

\subsection{Relationship Contents}

The activities carried out together with the romantic partners were the dimensions that appeared in the responses of parents' perceptions as something that distinguished the two generations, in addition to the existence of the similarities. A common difference was the intensity of interaction and communication in the romantic relationship. The second generation could easily communicate anywhere and anytime with the existence of technology in the form of cellphones and internet. Likewise, the interactions in recreational places or entertainment venues were more commonly carried out by the second generation, so that the romantic relationships of the second-generation adolescents were more free and open.

This situation is different from the one experienced by the first generation while they were in romantic relationship. The outside-home interaction was limited in the school environment and by communication through letters. With the development of technology, it was easier for the second generation to access and get some influence from foreign cultures. This condition, if supported by the attitude of Balinese tribes at present who tended to provide flexibility and minimal supervision, was considered very likely to be a factor supporting the couple activities that were considered improper before marriage, as reported by the first generation when differentiating the romantic relations activities from the two generations.

Physical contact in romantic relationship was one of the differences that were often mentioned by Balinese parents. They perceived that the second-generation romantic relationship tended to lead them to more intimate physical contact, which was described as a vulgar, very open, and very free, and caused the widespread of the phenomena of extramarital pregnancy and early marriage. This physical contact was perceived to be related to the reduction in fear of God and parents, and a sense of shame which in the past had become values that were considered important for regulating behavior in society.

The first generation of Balinese tribe also observed a shift in the role type in a romantic relationship. Women of the first generation were in a position to passively express feelings to their romantic partners, while in the second generation, the roles of men and women in a relationship tended to be more egalitarian. Balinese teenage girls of the second generation tended to be more assertive when establishing romantic relationships.

The similarity of the two generations identified by the first generation of Balinese tribe was the activity of visiting the couples' home and getting acquainted with the parents of the romantic partners. Despite the similarity, in fact, there was also a difference about this activity. In the romantic relationship of the first generation, it was natural for men to visit 
women's houses, but in the second generation, there were also found the women who visited the men's homes.

The Balinese teenage participants who had romantic relationship experience, on the other hand, reported a number of activities with romantic partners which are recreational and academic. Besides, they also reported other activities such as visitting the partners' home and having physical contact, from holding hands (the most frequent contact reported), hugging, kissing, touching, and having sex (the least frequent contact reported). They interacted fairly intensively through meetings at school and outside of school, and could communicate at any time desired with mobile phone facilities.

\subsection{Partner selection}

The differences between generations in the selection of romantic partners were revealed from the perceptions of parents who reported that when they first started romantic relationships, caste factors and matchmaking were common things to consider. This factor was less considered by the adolescents in the second generation in choosing their romantic partners. Another consideration that also arouse when the first generation began to establish romantic relationships was the idea of the importance of economic readiness which was considered to be one of the cornerstones when choosing a partner. It was related to the condition whether or not the partner possessed a job. This was in line with the purposes of the relationship that were to have a serious long-term relationship and to find a partner in life. This consideration was not considered important for the second generation. Adolescents of the second generation tended to easily change partners regardless of the consideration of castes and matchmaking which were rarely done and did not think about the partners' economic readiness for the relationship with a clearer goal.

In the selection of romantic partners, the second generation of Balinese tribe considered several things that could be grouped into a number of criteria, namely beautiful and attractive physical appearance possessed by the partners, positive personality characteristics which were considered as a priority, such as competencies in academics, sports, and arts; and similarities owned by the individual and their romantic partners in some ways, such as hobbies, character, religion, and caste.

Caste and religion were the considerations that also appeared in the experience of the first generation when choosing romantic partners. This condition led to arranged marriages set by the family which was common for the first generation. On the contrary, in this generation, it was perceived that arranged marriages were rare, and the adolescents had more freedom to choose and changed partners as they wished.

\subsection{Quality of relationships}

The first generation did not reveal much about how they perceived the quality of romantic relationship. The thing that could be assumed as the dimension of the quality of relationship from the first generation was that the relationship which was more serious and responsible, involved deep feelings and generally aimed to really get to know each other more closely, and even to find a spouse. This was assumed to be a form of romantic related experience that was more positive than the experience of second-generation adolescents. This condition was considered difficult to be found in adolescent relationships in the second generation which were perceived to be trial and error, and only fulfilled the curiosity. This romantic relationship often brought adverse effects to the adolescents who underwent the relationships in improper way and crossed the limit of norms in the society.

The dimension of the quality of relationships revealed by the adolescents of the second generation were more specifically reported from the positive and negative experiences. Many positive experiences revealed were related to the increasing academic motivation, the 
fulfillment of companionship needs, the availability of opportunities to get to know the characteristics of the opposite sex more closely and the opportunities for better personal growth. On the opposite, the negative experiences were generally related to the tendency of emotion and negative thoughts that caused discomfort when conflicts arouse or in the form of suspicion which gave an impact of the reduced motivation to learn. In addition, the setbacks in academic things were also influenced by the difficulty of managing time in everyday life which was dominated by activities with the romantic partners.

\subsection{Cognitive and emotional process}

The cognitive and emotional process was revealed as one dimension of romantic relationship which was related to the consideration of the cause and effect of the behaviors when establishing romantic relationship, as well as the behaviors formed by the emotions in the romantic relationship. The second-generation adolescents were perceived to be less concerned about the effects of their romantic behaviors or activities which were considered to be detrimental to themselves, their partners, and families. These behaviors, especially those that were intimate, very open, and tended to cross the limits of properness in the community. This was inseparable from the depletion of shame, respect, fear of parents and social sanctions in society. The second-generation adolescents tended not to consider the values that existed in the society and they might not believe that these values were important for regulating their behaviors. It is different from the first generation who identified the existence of shame, fear of God and parents, even the view that dating was taboo for some people, which led to romantic related behaviors that were politer and did not violate the norms in the society.

The experience of adolescents in romantic relationships, especially in the dimension of cognitive and emotional process, emphasized how they perceived the relationship and the emotions that were caused by the relationship. The adolescents in the second generation perceived their romantic relationship as a form of relationship that brought a change towards maturity and a more meaningful daily life. Their romantic relationship involved a feeling of deep affection and a commitment to a successful relationship. The relationship could also cause the feelings of pleasure and satisfaction, sometimes the feeling of anxiety to be betrayed and parted, and the fear that arouse when the relationship was carried out without being known by the parents.

\subsection{Interpersonal context}

How parents behaved and responded to their sons' and daughters' romantic relationship was one of the factors perceived by the first generation to influence the differences between the two generations. The first generation experienced the strict parental supervision and the grumpy characteristics of the parents who would be angry knowing their children when having a romantic relationship, especially when they were at school. On the contrary, the parents of the second-generation adolescents were more open to accept the teenagers' social development, which caused the parents to soften their grumpy reaction when their adolescents were in romantic relationship. The aspect of parental supervision was one of the important things that were perceived to begin to diminish at present. This was especially true when the adolescents did not live with their parents because they studied in a different area from their families.

The influence of parents on romantic relationship in the second generation arouse in the context of the presence or absence of permission from parents to establish romantic relationship and parental responses to this relationship. The group of teenagers who hid their romantic relationship reported that it was done because the parents did not allow them to date when they were still in school and were still considered too young. Whereas, the adolescents who were open to publish their relationship reported that they got a positive response from the 
parents, introduced their partners to the parents, and were asked to stay focused on education and behave well by their parents. Some adolescents did not consider the permission and response of parents because their parents never interfered with their personal matters and tended to give freedom to them to act according to their own desires and needs.

\subsection{Development of technology and social media}

The ease of communication and the increasing intensity of interaction in the romantic relationship of adolescent in the second generation, as well as increasingly bold and open attitudes and behavior, were perceived by the first generation as a result of technological developments. Internet access that was very easy to get caused them to quickly receive and absorb the influence of foreign culture. In addition the sophisticated communication tools enabled them to communicate with their partners wherever and whenever. Social media also became a medium for them to show their relationship and express their feelings to their partners.

The adolescents made use of the technological developments in the romantic relationship, and it could be indirectly concluded that it was also one of the activities they conducted with the romantic partners. The communication which was carried out by using mobile phones and social media could take place at any time without being limited by time and space. The adolescents of the second generation reported that the influence of foreign culture caused a tendency for dating behavior that was not good and was not in accordance with the norms of the society. However, it was not explicitly revealed that this was the impact of the development of technology existed in the adolescents' life.

The results of the study showed that there were a number of differences in addition to the similarity of perceptions and experiences in the romantic relationship dimensions of the two generations of Balinese tribe. The age of involvement in the romantic relationship seemed to be shifting to be younger. It was followed by the activities conducted by the couples of the second generation that were more various, up to the intimate physical contact which led to the increase of premarital pregnancies and early marriage phenomena. The adolescents of the second generation tended to be easier to start and end a relationship as well as change partners. When viewed from the results of this study, more than $60 \%$ of Balinese adolescents at present aged 14-17 years were familiar with romantic relationships with various frequency and duration, and the rest reported never having a romantic relationship. The reasons expressed, among others, were the parents who did not permitted the relationships and the desire to focus on education. These reasons were similar to what were revealed by the first generation of Balinese tribe when explaining the reasons for the small number of adolescents of that generation establishing romantic relationship. The adolescents in collectivistic societies tend to have a lack form of romantic involvement, show a low romantic experience, and have few close romantic relationships when compared to the adolescents in individualistic cultures [4]. It was related to Asian cultural values that has more consideration for starting a relationship that is related to the family ties and family arrangements, especially the consideration given by parents.

Though some of the Balinese adolescents from the second generation did not get any permission from their parents to have a romantic relationship, they still had some experience in romantic relations and underwent it secretly. This was done to avoid the anger from parents. The adolescents, especially in the early period, in certain cultures, such as Latin society, are more closely watched when interacting with the opposite sex and when they establish adolescent romantic relationship, they tend to keep it a secret from their family because of their fear of being forced to end the relationship [8]. Markus and Kitayama [4] argues that by delaying romantic involvement, Asian families extend the period in which parents act as the 
main influence on their offspring. On the contrary, by allowing teenagers to act as free agents in their romantic involvement, western families facilitate youth individualism.

The activities with the partners which were commonly carried out by the first Balinese generation appeared to be quite different from what were usually done by second-generation adolescents. This was understood as the impact of the influence of the social environment development and it was supported by the technological advances in terms of communication and access to the influx of external cultural influences or modernization. Dhariwal and Connolly [9] revealed that adolescents who gained western influence had more romanticrelated activities. The influence of western media consumption was also considered to cause more permissive friendships, wider networks and intimate communication. The influx of western culture into Balinese society is a well-known condition, considering that Bali is a foreign tourist destination for decades and in the present time, the influence of western culture is indirectly absorbed more through the technological developments. In the other hand the use of the internet is seen as beneficial for improving the quality of romantic relationships and is useful for maintaining relationships or looking for potential romantic partners [10].

The change of the role type in romantic relation was one interesting thing that differentiated the two generations. Teenage girls in the first generation were more passive in expressing feelings. It is different from the attitude of today's young women who were more assertive and egalitarian compared with the role of adolescent boys. In the culture of eastern communities themselves, parents impose more control over girls than boys. This causes the teenage boys to be more comfortable with expressions of intimate feelings compared to teenage girls [11].

In the context of choosing partners in romantic relationship, there were norms, values, rules or habits that were transmitted in the culture. The traditional attributes that the couples possessed became a priority in partner selection to support traditional family expectations and self-construl interdependence which was revealed by Lalonde, et al. [12] in Asian participants. It is in line with the selection of partner which prioritize religious values [13] which become a large part of a particular culture. The second generation of Balinese tribes, in addition to the fact of prioritizing physical appearance, competence, personality, similarities and hobbies, also considered religion and caste in choosing a romantic partner. The first generation of Balinese tribe, in addition to the consideration of economic readiness, expressed the same thing, that was the existence of arranged marriage which was set by the families considering the similarity of the caste and religion.

Dhariwal and Connolly [9] suggest that adolescents in different cultural contexts can feel the different levels of autonomy given by the parents regarding romantic partner selection. In some cultures, including western societies, individuals usually start dating during adolescence, often with great freedom in choosing their romantic partners and less pressure to develop committed relationships.

In term of the quality of a romantic relationship, the two generations of Balinese tribe showed a difference. The first-generation romantic relationship involved deeper feelings which led to more serious forms of relationships, such as marriages. This relationship was carried out responsibly so that it was more positive than the experience of the adolescents of the second generation who established the romantic relationships only because of curiosity and led the relationships to the less irresponsible behaviors. On the other hand, Balinese adolescents in the second generation revealed that romantic relationships could bring more specific positive experiences, such as meeting companionship needs and providing motivation in the academic field. The negative experiences that was felt by them when having romantic relationships were more related to the emergence of emotions and negative thoughts that 
caused discomfort in the relationships, and the problems of learning motivation when experiencing conflicts in romantic relationship.

The first generation of Balinese tribe considered that Balinese adolescents did not think about the impact of intimate activities in their romantic relationship. It is different from their previous experiences when behaviors were still attached to shame, fear, respect, and fear of parents and God. The adolescents in the second generation were more interested in perceiving romantic relationship as a relationship that brought good influence in their lives and gave various emotional experiences that matured them personally. Deep feelings in romantic relationships were actually not only possessed by the first generation, but also became a part of the romantic journey of some Balinese tribe in the second generation. It is similar to the commitment in a relationship that was not only important for the first generation but also for the second generation.

The experience of the two generations of Balinese tribe regarding romantic relationship presented the role of parents as part of the interpersonal context. In the collective culture, parents could be the main force of socialization for teen romance, the transmission of cultural knowledge about romantic relationship, emphasis on the respect for families and the importance of responsibility [14]. Romantic relationships undertaken by adolescents in certain situations can lead to the emergence of risk behaviors that can have a negative impact on the future of adolescents. Parents play an important role in preventing risky behavior in adolescents. This relates to adolescent openness and control carried out by parents so that parents have knowledge about the activities carried out by their children. Confident parenting and a close parent and child relationships can protect adolescents from risky behavior [15].

\section{CONCLUSIONS}

The results of data analysis of the two generations which were discussed from the viewpoint of experience and perceptions of the two Balinese generations showed that each dimension of romantic relationship was related to one another. It interacted with the interpersonal context, in this case the role of parents, and the existence of modernization through the influence of technological developments. It gave an overview of the differences and similarities in the experience of romantic relationship of the two generations. The cultural context of individualism and collectivism was used in discussing the research results and it enabled the researchers and readers to understand the cultural expectations of romantic relationship in a community. In addition, this approach also led the researchers to see whether there was a shift in the view of Balinese culture from collectivistic to individualistic, although to reach this conclusion certainly requires a lot more comprehensive research.

Since the perceptions of the parents were based on the generalizable experience and observations, it could be concluded that in some parts of the romantic relationship dimensions of second-generation adolescents were less confirmed by the experience of the adolescents. The aspects that according to the parents differed between these generations, seemed to be identified as aspects that appeared at present based on experiences reported by the adolescents. This condition was very possible to happen because the first generation that currently played a role as parents transmitted values or beliefs about how these relationships should be done. Whereas, the changes in the interpersonal context that rouse the shift in parenting patterns showed a tendency towards a change in the attitude of the first generation to view romantic relationships that were quite different from their past experiences. What they perceived as something different between generations were the results of more general observations for example from the phenomenon of present teenage relationship. Regarding the method used in 
this study, the use of survey methods used for exploration would be better to be followed by in-depth interviews or focused group discussion (FGD) to explore deeper romantic relationships experiences of the two generations.

The implications of this study lead to the need for a more comprehensive study of romantic relationship in a cultural context given that the characteristics of Balinese culture are in contrast to the tendency of romantic relationship change at present. This is related to the study of the interplay of the changes in Balinese cultural values and changes in the romantic relationship of each generation of Balinese people. In this case, the broader direction of research can investigate what and how the transmission of values is related to the romantic relationship of Balinese people so that they discover the concept of contemporary Balinese society romantic relationship.

\section{REFERENCES}

[1] W. A. Collins, "More than myth: The developmental significance of romantic relationships during adolescence," J. Res. Adolesc., vol. 13, no. 1, pp. 1-24, 2003.

[2] W. A. Collins, D. P. Welsh, and W. Furman, "Adolescent Romantic Relationships," Annu. Rev. Psychol., vol. 60, no. 1, pp. 631-652, 2009.

[3] D. van de Bongardt, R. Yu, M. Deković, and W. H. J. Meeus, "Romantic relationships and sexuality in adolescence and young adulthood: The role of parents, peers, and partners," Eur. J. Dev. Psychol., vol. 12, no. 5, pp. 497-515, 2015.

[4] Z. H. Li, J. Connolly, D. Jiang, D. Pepler, and W. Craig, "Adolescent romantic relationships in China and Canada: A cross-national comparison," Int. J. Behav. Dev., vol. 34, no. 2, pp. 113-120, 2010.

[5] T. A. Chandalia, "Modernization and Social Change among Adolescence OBJECTIVES : HYPOTHESIS : Tools :," vol. 2, no. 3, 2015.

[6] J. Sun and X. Wang, "Value differences between generations in China: A study in Shanghai," J. Youth Stud., vol. 13, no. 1, pp. 65-81, 2010.

[7] R. J. Mccammon, "Generations , Cohorts , and Social Change," no. January 2003, 2014.

[8] L. F. O'Sullivan and H. F. L. Meyer-Bahlburg, "African-American and Latina InnerCity Girls' Reports of Romantic and Sexual Development," J. Soc. Pers. Relat., vol. 20, no. 2, pp. 221-238, 2003.

[9] A. Dhariwal and J. Connolly, "Romantic Experiences of Homeland and Diaspora South Asian Youth: Westernizing Processes of Media and Friends," J. Res. Adolesc., vol. 23, no. 1, pp. 45-56, 2013.

[10] L. A. Bagley and C. Kimberly, "Technology use and its association with romantic relationships," Contemp. Perspect. Fam. Res., vol. 11, pp. 217-236, 2017.

[11] S. M. Moore and C. Leung, "Romantic beliefs, styles, and relationships among young people from Chinese, Southern European, and Anglo-Australian backgrounds," Asian J. Soc. Psychol., vol. 4, no. 1, pp. 53-68, 2001.

[12] R. N. Lalonde, M. Hynie, M. Pannu, and S. Tatla, "The role of culture in interpersonal relationships: Do second generation South Asian Canadians want a traditional partner?,” J. Cross. Cult. Psychol., vol. 35, no. 5, pp. 503-524, 2004.

[13] R. P. Bernarte, A. K. L. Jalandra, J. R. F. Jarquio, and M. E. Sanggo, "The criteria of love: Trait desirability of filipino youth on mate selection," Asia Pacific J. Multidiscip. Res., vol. 4, no. 3, pp. 10-17, 2016.

[14] I. Seiffge-Krenke and J. Connolly, "Adolescent romantic relationships across the 
globe: Involvement, conflict management, and linkages to parents and peer relationships," Int. J. Behav. Dev., vol. 34, no. 2, p. 97, 2010.

[15] S. Kapetanovic, T. Skoog, M. Bohlin, and A. Gerdner, "Aspects of the parentadolescent relationship and associations with adolescent risk behaviors over time," $J$. Fam. Psychol., vol. 33, no. 1, pp. 1-11, 2019. 